\title{
Rough Approximations under Level Fuzzy Sets
}

\author{
W.-N. Liu J.T. Yao Y.Y.Yao \\ Department of Computer Science, University of Regina \\ Regina, Saskatchewan, Canada S4S 0A2 \\ E-mail: [liuwe200, jtyao, yyao]@cs.uregina.ca
}

\begin{abstract}
The combination of fuzzy set and rough set theories lead to various models. Functional and set approaches are two categories based on different fuzzy representations. In this paper, we study rough approximations based on the notion of level fuzzy sets. Two rough approximation models, namely $\alpha$-level rough set and $\beta$-level rough set, are proposed. It shows that $\beta$-level fuzzy rough set model can approximate a fuzzy set at different precisions.
\end{abstract}

\section{Introduction}

The distinct and complementary fuzzy set theory [15] and rough set theory [7] are generalizations of classical set theory. Attempts to combine these two theories lead to new notions $[2,10,13]$. The combination involves three types of approximations, i.e., approximation of fuzzy sets in crisp approximation spaces, approximation of crisp sets in fuzzy approximation spaces, and approximation of fuzzy sets in fuzzy approximation spaces [13].

The construction of fuzzy rough sets can be classified into two approaches, namely, functional approach and set approach. The first one formulates the lower and upper bounds with fuzzy membership functions. These formulas express the logical relations that lower and upper bounds must abide in approximation spaces [10].

The second approach [13] combines rough and fuzzy sets based on the cutting of fuzzy sets or fuzzy relations. When a fuzzy set is represented by a family of crisp subsets ( $\alpha$-level sets), these $\alpha$-level sets can be approximated by equivalence relations in rough sets. A fuzzy relation can also be approximated by a family of equivalence relations ( $\beta$-level sets). This family defines a family of approximation spaces. The new rough sets are based on these approximation spaces.

A third approach of the combination of fuzzy sets and rough sets can be considered by introducing the concept of level fuzzy sets. It has been argued that benefits do exist in the use of level fuzzy sets over level sets $[1,9,11]$.

The present study examines some of the fundamental issues of the combination from the perspective of level fuzzy sets. The properties of $\alpha$-level fuzzy sets and $\beta$-level fuzzy sets will be introduced in the next section. The models of $\alpha$-level rough set and $\beta$-level rough set are studied. We discuss the properties of these models in Section 3. 


\section{$2 \quad$ Fuzzy Rough Sets and Level Fuzzy Sets}

We review the concept of fuzzy rough sets and level fuzzy sets. The properties of level fuzzy sets are also discussed.

\subsection{Fuzzy Rough Sets}

Many views of fuzzy rough sets exist. We adopt the notion of Radzikowska and Kerre [10], which absorbs some earlier studies [3, 4,6] in the same direction. Let (U,R) be a fuzzy approximation space and $\tilde{P}(U)$ be the set of all fuzzy sets. For every $A \in \tilde{P}(U), \operatorname{apr}(A)=\left(\underline{a p r}_{R}(A), \overline{a p r}_{R}(A)\right)$ where

$$
\begin{aligned}
& \frac{a p r}{R}(A)(x)=\inf _{y \in U} I(R(x, y), A(y)) \\
& \overline{a p r}_{R}(A)(x)=\sup _{y \in U} T(R(x, y), A(y)) .
\end{aligned}
$$

${ }_{\operatorname{apr}}(A)$ and $\left.\overline{\operatorname{apr}}_{R}(A)\right)$ define the lower and upper approximations of a fuzzy set A respectively. They are constructed by means of an implicator I and a t-norm T. Equation 1 indicates that, for any $x \in U$, its membership degree is determined by looking at the elements resembling $x$, and by computing to what extent $y$ is contained in a fuzzy set A. Equation 2 indicates that the membership degree of $x$ is determined by the overlap between $y$ and A.

\subsection{Level Sets and Level Fuzzy Sets}

Let $\mathrm{A}$ be a fuzzy set defined in universe $\mathrm{U}$, and $\alpha \in(0,1]$. The $\alpha$-level set or $\alpha$-cut of $\mathrm{A}$ is a crisp subset of $\mathrm{U}$ defined by

$$
A_{\alpha}(x)=\left\{\begin{array}{l}
1 \text { if } A(x) \geq \alpha \\
0 \text { otherwise }
\end{array} ;\right.
$$

the $\alpha$-level fuzzy set or fuzzy $\alpha$-cut of $\mathrm{A}$ is characterized by

$$
\tilde{A}_{\alpha}(x)=\left\{\begin{array}{ll}
A(x) & \text { if } A(x) \geq \alpha \\
0 & \text { otherwise }
\end{array} .\right.
$$

Based on above definitions, we can conclude that $\alpha$-level fuzzy sets are obtained by reducing parts of fuzziness or information holding in the original fuzzy sets.

Let $\mathrm{R}$ be a fuzzy similarity relation on $\mathrm{U}$, and $\beta \in(0,1]$. The $\beta$-level set or $\beta$-cut of $\mathrm{R}$ is an equivalence relation on $\mathrm{U}$ defined by

$$
R_{\beta}(x, y)=\left\{\begin{array}{l}
1 \text { if } R(x, y) \geq \beta \\
0 \text { otherwise }
\end{array} ;\right.
$$

the $\beta$-level fuzzy set or fuzzy $\beta$-cut of $\mathrm{R}$ is characterized by

$$
\tilde{R}_{\beta}(x, y)=\left\{\begin{array}{ll}
R(x, y) & \text { if } R(x, y) \geq \beta \\
0 & \text { otherwise }
\end{array} .\right.
$$


Both $\alpha$-level set and $\beta$-level set are called level sets; correspondingly, both $\alpha$-level fuzzy set and $\beta$-level fuzzy set are called level fuzzy sets. The symmetry between level sets and level fuzzy sets indicates that the properties of level fuzzy sets are a fuzzy counterpart of the ones of level sets.

Property 1. $\forall \alpha \in[0,1], \forall x \in U, \tilde{A}_{\alpha} \subseteq A$ and $\operatorname{supp}_{\tilde{A}} \subseteq \operatorname{supp} A$ $\forall \alpha_{1}, \alpha_{2} \in[0,1], \alpha_{2} \geq \alpha_{1} \Rightarrow \tilde{A}_{\alpha_{2}} \subseteq \tilde{A}_{\alpha_{1}}$ and supp $\tilde{A}_{\alpha_{2}} \subseteq \operatorname{supp} \tilde{A}_{\alpha_{1}}$.

Property 1 indicates that $\alpha$-level fuzzy sets are monotonic with respect to fuzzy set inclusion. The supports of $\alpha$-level fuzzy sets are monotonic with respect to set inclusion.

Property 2. For every $\beta \in[0,1]$, each $\tilde{R}_{\beta}$ of similarity relation $\mathrm{R}$ is a similarity relation on $\mathrm{U}$, i.e. $\tilde{R}_{\beta}$ satisfies reflexive, symmetric and sup-min transitive conditions.

Proof.

It is only necessary to verify that $\tilde{R}_{\beta}$ satisfies the sup-min transitive condition. when $R(x, y) \geq \beta$,

$\tilde{R}_{\beta}(x, y) \geq \sup _{z \in U}\{\min \{R(x, z), R(z, y)\}\} \geq \sup _{z \in U}\left\{\min \left\{\tilde{R}_{\beta}(x, z), \tilde{R}_{\beta}(z, y)\right\}\right\}$ when $R(x, y)<\beta, \tilde{R}_{\beta}(x, y)=0$

$\beta>R(x, y) \geq \sup _{z \in U}\{\min \{R(x, z), R(z, y)\}\}$

which means that $\forall z \in U, R(x, z)<\beta$ or $R(z, y)<\beta$ i.e.

$\forall z \in U, \tilde{R}_{\beta}(x, z)<\beta$ or $\tilde{R}_{\beta}(z, y)<\beta$ i.e.

$\forall z \in U, \min \left\{\tilde{R}_{\beta}(x, z), \tilde{R}_{\beta}(z, y)\right\}=0$

We still have the result: $\tilde{R}_{\beta}(x, y) \geq \sup _{z \in U}\left\{\min \left\{\tilde{R}_{\beta}(x, z), \tilde{R}_{\beta}(z, y)\right\}\right\} \square$

In fuzzy approximation space, basic granules of knowledge can be represented by similarity classes for each element in $U$ [12]. The size of the support of every similarity class is used to measure the granularity of the class. More precisely, the similarity class for $x \in U$, denoted $R_{x}$, is a fuzzy set in $\mathrm{U}$ characterized by the membership function: $R_{x}(y)=R(x, y)$. The similarity class for $x \in U$ determined by $\tilde{R}_{\beta}$, denoted $R_{x}^{\beta}$, is characterized by the membership function: $R_{x}^{\beta}(y)=\tilde{R}_{\beta}(x, y)$.

Property 3. $\forall \beta, \beta_{1}, \beta_{2} \in[0,1], \forall x \in U, \operatorname{supp} R_{x}^{\beta} \subseteq \operatorname{supp} R_{x}$ and $\beta_{2} \geq \beta_{1} \Rightarrow R_{x}^{\beta_{2}} \subseteq$ $R_{x}^{\beta_{1}}$

Property 3 indicates that the $\beta$-level fuzzy sets of a similarity relation form a nested sequence of similarity relations. The bigger level $\beta$, the finer the similarity classes determined by $\tilde{R}_{\beta}$. Property 2 and 3 justify that $\beta$-level fuzzy sets are a fuzzy counterpart of $\beta$-level sets. The sequence of fuzzy relations coincides with the partition tree [5] constructed by $\beta$-level sets.

\section{$3 \quad$ Level Fuzzy Sets based Fuzzy Rough Sets}

Any fuzzy set can be decomposed into a family of $\alpha$-level sets and a family of $\alpha$-level fuzzy sets. Any fuzzy relation can also be decomposed into a family of 
$\beta$-level sets and a family of $\beta$-level fuzzy sets. In Section 3.1, the reference set $\mathrm{A}$ in $\left(\operatorname{apr}_{R}(A), \overline{a p r}_{R}(A)\right)$ is replaced with its $\alpha$-level fuzzy set. In Section 3.2, fuzzy relation $\mathrm{R}$ in $\left(\underline{a p r}_{R}(A), \overline{a p r}_{R}(A)\right)$ is substituted with its $\beta$-level fuzzy set. Two new fuzzy rough sets are obtained. We examine their properties and briefly demonstrate how level fuzzy sets simplify the computation of fuzzy rough sets.

\section{1 $\alpha$-level Fuzzy Rough Set Model}

Consider the approximation of an $\alpha$-level fuzzy set of the reference set A, $\alpha \in(0,1]$, in the fuzzy approximation space (U,R). The fuzzy rough set $\left(\underline{\operatorname{apr}} \underline{R}_{R}\left(\tilde{A}_{\alpha}\right), \overline{\operatorname{apr}}_{R}\left(\tilde{A}_{\alpha}\right)\right)$ :

$$
\begin{aligned}
& \frac{a p r}{R}\left(\tilde{A}_{\alpha}\right)(x)=\inf _{y \in U} I\left(R(x, y), \tilde{A}_{\alpha}(y)\right) \\
& \overline{a p r}_{R}\left(\tilde{A}_{\alpha}\right)(x)=\sup _{y \in U} T\left(R(x, y), \tilde{A}_{\alpha}(y)\right)
\end{aligned}
$$

is called the $\alpha$-level fuzzy rough sets of A. For the family of $\alpha$-level fuzzy sets, we obtain a family of $\alpha$-level fuzzy rough sets.

Property 4. If fuzzy implicator I is right monotonic, and implicator I and t-norm $\mathrm{T}$ are continuous, then $\forall \alpha, \alpha_{1}, \alpha_{2} \in[0,1]$,

$\underline{\operatorname{apr}}_{R}(A) \supseteq \underline{\operatorname{apr}}_{R}\left(\tilde{A}_{\alpha}\right)$ and $\overline{a p r}{ }_{R}(A) \supseteq \overline{\operatorname{apr}}_{R}\left(\tilde{A}_{\alpha}\right)$;

$\alpha_{1} \leq \alpha_{2} \Rightarrow \underline{a p r}_{R}\left(\tilde{A}_{\alpha_{1}}\right) \supseteq \underline{a p r}_{R}\left(\tilde{A}_{\alpha_{2}}\right)$ and $\overline{a p r}_{R}\left(\tilde{A}_{\alpha_{1}}\right) \supseteq \overline{\operatorname{apr}}_{R}\left(\tilde{A}_{\alpha_{2}}\right)$.

Property 4 indicates that $\alpha$-level fuzzy rough sets are monotonic with respect to fuzzy set inclusion. The property is similar to that of $\alpha$-level rough sets. However, we have to concede that, unlike $\alpha$-level rough sets [13], there is no guarantee that $\operatorname{apr}_{R}\left(\tilde{A}_{\alpha}\right)$ will be $\alpha$-level fuzzy set of some fuzzy set. The conclusion is the same with $\overline{a p r}_{R}\left(\tilde{A}_{\alpha}\right)$. We can not say that the family of $\left(\underline{a p r}{ }_{R}\left(\tilde{A}_{\alpha}\right), \overline{a p r}_{R}\left(\tilde{A}_{\alpha}\right)\right)$ define $\left.\underline{\operatorname{apr}}_{R}(A), \overline{a p r}_{R}(A)\right)$.

Conversely, we notice that the computation of $(\operatorname{apr}(A), \overline{\operatorname{apr}}(A))$ can be divided into the evaluation of implication $\mathrm{I}(\mathrm{R}(\mathrm{x}, \mathrm{y}), \mathrm{A} \overline{(\mathrm{y})})$, the evaluation of cojunction $\mathrm{T}(\mathrm{R}(\mathrm{x}, \mathrm{y}), \mathrm{A}(\mathrm{y}))$, and the evaluation of infimum and supremum. By the property of implicator $\mathrm{I}$, if $\mathrm{A}(\mathrm{y})=0$, the value of $\mathrm{R}(\mathrm{x}, \mathrm{y})$ alone determines the value of $\mathrm{I}(\mathrm{R}(\mathrm{x}, \mathrm{y}), \mathrm{A}(\mathrm{y}))$ and $T(R(x, y), A(y)) \equiv 0$. There are less elements participating in the computation of $\left(\underline{a p r}_{R}(A), \overline{a p r}_{R}(A)\right.$ by replacing the fuzzy set $\mathrm{A}$ with its $\alpha$-level fuzzy set. From a practical view, $\alpha$-level fuzzy sets simplify the computation of fuzzy rough sets. The total saved running time is in proposition to level $\alpha$.

\section{$3.2 \quad \beta$-level Fuzzy Rough Set Model}

The family of $\beta$-level fuzzy sets of fuzzy relation $\mathrm{R}$ defines a family of approximation spaces: $\left(U, \tilde{R}_{\beta}\right), \beta \in(0,1]$. For a $\beta \in(0,1]$, the fuzzy rough set 
$\left(\underline{a p r}_{\tilde{R}_{\beta}}(A), \overline{a p r}_{\tilde{R}_{\beta}}(A)\right):$

$$
\begin{aligned}
& \frac{a p r}{\tilde{R}_{\beta}}(A)(x)=\inf _{y \in U} I\left(\tilde{R}_{\beta}(x, y), A(y)\right) \\
& \overline{a p r} \tilde{R}_{\beta}(A)(x)=\sup _{y \in U} T\left(\tilde{R}_{\beta}(x, y), A(y)\right)
\end{aligned}
$$

are called the $\beta$-level fuzzy rough sets of A. With respect to a fuzzy approximation space, we obtain a family of $\beta$-level fuzzy rough sets. The following properties can be verified easily:

Property 5. If the fuzzy implicator I is a continuous R-implicator based on a continuous t-norm T, then $\forall A \in \tilde{P}(U), \underline{a p r}_{\tilde{R}_{\beta}}(A) \subseteq A \subseteq \overline{a p r}_{\tilde{R}_{\beta}}(A)$.

Property 6. If fuzzy implicator I is left monotonic, and I and t-norm $\mathrm{T}$ are continuous, then

$\underline{a p r}_{R}(A) \subseteq \underline{a p r}_{\tilde{R}_{\beta}}(A)$ and $\overline{a p r}_{R}(A) \supseteq \overline{a p r}_{\tilde{R}_{\beta}}(A) ;$

$\beta_{1} \leq \beta_{2} \Rightarrow \underline{a p r} \tilde{R}_{\beta_{1}}(A) \subseteq \underline{a p r} \tilde{R}_{\beta_{2}}(A)$ and $\overline{a p r} \tilde{R}_{\beta_{1}}(A) \supseteq \overline{a p r}_{\tilde{R}_{\beta_{2}}}(A)$.

Property 6 indicates that $\beta$-level fuzzy rough sets are monotonic with respect to the refinement of fuzzy relations. Coarse similarity classes usually lead to a 'coarse' approximation with a great misclassification error, whereas smaller similarity classes usually lead to a 'fine' approximation with a less misclassification error. Property 5 and 6 also indicate that a nested sequence of $\beta$-level fuzzy sets can lead to hierarchical rough approximations. The approximating precision can be controlled by adjusting level $\beta$. However, unlike $\beta$-level rough sets, there is no guarantee that $\left(\underline{\operatorname{apr}} \tilde{R}_{\beta}(A), \overline{a p r}_{\tilde{R}_{\beta}}(A)\right)$ is a level fuzzy set of $(\underline{a p r}(A), \overline{a p r}(A))$.

Similar with $\alpha$-level fuzzy rough sets, $\beta$-level fuzzy sets can eliminate part of the computation of lower approximation and upper approximation. The reason is that if $\mathrm{R}(\mathrm{x}, \mathrm{y})=0$, then $I(R(x, y), A(y)) \equiv 1$ and $T(R(x, y), A(y)) \equiv 0$. The total saved running time is in proposition to level $\beta$.

The $\beta$-level sets of similarity relations form a nested sequence of equivalence relations. Let $A \subseteq U$, for each $\beta$-level sets of $\mathrm{R}, R_{\beta}$, a crisp $\beta$-level rough set $\left.{ }_{(a p r} R_{\beta}(A), \overline{a p r} R_{\beta}(A)\right)$ satisfies all the properties of rough set.

Property 7. $\forall \beta_{1}, \beta_{2} \in[0,1], \beta_{1} \leq \beta_{2} \Rightarrow \underline{a p r}_{R_{\beta_{1}}}(A) \subseteq \underline{a p r}_{R_{\beta_{2}}}(A)$ and $\overline{a p r}_{R_{\beta_{1}}}(A) \supseteq$ $\overline{a p r}_{R_{\beta_{2}}}(A)$.

\section{Conclusions}

We introduce a new approach to the combination of fuzzy sets and rough sets. The combination is based on level fuzzy sets. We propose both the $\alpha$-level fuzzy rough set model and the $\beta$-level fuzzy rough set model. It provides a new perspective to the theories of fuzzy sets and rough sets. Similar to the $\alpha$-level rough sets and the $\beta$-level rough sets, some useful properties are examined. The $\beta$-level 
fuzzy rough sets may approximate a fuzzy set at different precisions by choosing different $\beta$-level fuzzy sets of a similarity relation.

Level fuzzy sets may reduce the information that implication and cojunction have to work with. This may lead to a simple computation. The trade-offs between approximating precision and computational efficiency are under examination. Decision-theoretic Rough Set theory [14] may play an important role in selecting proper $\alpha$ and $\beta$ level values.

\section{References}

1. Baets, B.D., Kerre, E., "The Cutting of Compositions", Fuzzy Sets and Systems, Vol.62, pp.295-309, 1994.

2. Cornelis, C., Cock, M.D. and Kerre, E.E., "Intuitionistic Fuzzy Rough Sets: At the Crossroads of Imperfect Knowledge", Expert Systems, Vol.20, No.5, pp.260270, Nov., 2003.

3. Dubois, D. and Prade, H., "Putting rough sets and fuzzy sets together", Intelligent Decision Support: Handbook of Applications and Advances of the Rough Sets Theory, Slowinski, R., (Ed.), Kluwer Academic Publishers, Boston, pp.203-222, 1992.

4. Dubois, D. and Prade, H., "Rough Fuzzy Sets and Fuzzy Rough Sets", International Journal of general systems, 17, pp.191-209, 1990

5. Dubois, D., Prade, H., "Fuzzy Sets and Systems: Theory and Applications", Academic Press, New York, 1980.

6. Nakamura, A., "Fuzzy Rough Sets", Notes on Multiple-Valued Logic in Japan, 9, pp.1-8, 1988

7. Pawlak, Z., "Rough Sets: Theoretical Aspects of Reasoning About Data", Kluwer Academic Publishers, Dordrecht, 1991.

8. Radecki, T., "A Model of a Document Retrieval System based on the Concept of a Semantic Disjunctif Normal Form", Kybernetes, Vol.10, pp.35-42, 1981.

9. Radecki, T., "Level Fuzzy Sets", J. Cybernet, Vol.7, pp.189-198, 1977.

10. Radzikowska, A.M. and Kerre, E.E., "A Comparative Study of Fuzzy Rough Sets", Fuzzy Sets and Systems, Vol.126, pp.137-155, 2002.

11. Rembrand, B.R.B., Zenner and Rita M.M. DE Caluwe, "A New Approach to Information Retrieval Systems Using Fuzzy Expressions", Fuzzy Sets and Systems, Vol.17, pp.9-22, 1984.

12. Slowinski, R. and Vanderpooten, D., "A Generalized Definition of Rough Approximations Based on Similarity", IEEE Transactions on Knowledge and data engineering, Vol.12, No.2, pp.331-336, 2000.

13. Yao, Y.Y., "Combination of Rough and Fuzzy Sets Based on $\alpha$-Level Sets", Rough Sets and Data Mining: Analysis for Imprecise Data, Lin, T.Y. and Cercone, N. (Ed.), Kluwer Academic, Boston, MA, pp.301-321, 1997.

14. Yao, Y.Y., Wong, S.K.M, "A Decision Theoretic Framework for Approximating Concepts", International Journal of Man-machine Studies, Vol.37, No.6, pp.793809, 1992.

15. Zadeh, L., "Fuzzy Sets", Information and Control, Vol.8, pp.338-353, 1965. 